\title{
Aumento na expressão de telomerase por fibroblastos ocorre precocemente à expressão de alfa-actina de músculo liso por miofibroblastos no processo de remodelamento da pneumonia intersticial usual
}

\author{
Increased telomerase expression in fibroblasts occurs earlier \\ than myofibroblast alfa-smooth muscle actin expression in the \\ remodeling process of usual interstitial pneumonia
}

\author{
Daniel Reis Waisberg ${ }^{1}$, Osmar Bianchi da Silva ${ }^{1}$, Marcelo Junqueira Atanazio', \\ Edwin Roger Parra², João Valente Barbas Filho³, Vera Luiza Capelozzi ${ }^{4}$
}

\begin{abstract}
Waisberg DR, Silva OB, Atanazio MJ, Parra ER, Barbas Filho JV, Capelozzi VL. Aumento na expressão de telomerase por fibroblastos ocorre precocemente à Expressão de $\alpha$-actina de músculo liso por miofibroblastos no processo de remodelamento da pneumonia intersticial usual/ Increased telomerase expression in fibroblasts occurs earlier than myofibroblast $\alpha$-smooth muscle actin expression in the remodeling process of usual interstitial pneumonia. Rev Med (São Paulo). 2011;90(1):36-46.
\end{abstract}

\begin{abstract}
RESUMO: Objetivo: Identificar potenciais marcadores associados à expressão de telomerase em fibroblastos e de $\alpha$-actina de músculo liso ( $\alpha$-AMS) em miofibroblastos de pulmões de pacientes com fibrose pulmonar idiopática/pneumonia intersticial usual (FPI/ PIU). Métodos: Utilizamos cortes histológicos de 34 biópsias cirúrgicas de pulmão de pacientes com FPI, caracterizados, à histopatologia, pelo padrão de PIU. As expressões de telomerase por fibroblastos, de $\alpha$-AMS por miofibroblastos e tecidual de interleucina-4 (IL-4), de fator de crescimento transformador- $\beta$ (TGF- $\beta$ ) e de fator de crescimento de fibroblastos básico (bFGF) foram avaliados por imunohistoquímica e quantificadas pela técnica de contagem de pontos nas áreas pulmonares de colapso (COL), de fibrose mural (FM) e de faveolamento (FV). Resultados: A expressão de telomerase foi significativamente maior nas áreas de COL que nas áreas de FM e FV. O mesmo foi observado para a expressão de bFGF. Interleucina-4 e $\alpha$-AMS tiveram expressão significativamente maior nas áreas de FM. A expressão de TGF- $\beta$ foi maior nas áreas de COL e FV. Observamos uma associação positiva entre expressão de telomerase e bFGF nas áreas COL,
\end{abstract}

ABSTRACT: Objective: To identify potential markers associated with fibroblast telomerase and interstitial myfibroblast alpha-smooth muscle actin ( $\alpha$-AMS) expression in patients with idiopathic pulmonary fibrosis/usual intersticial pneumonia (IPF/UIP). Methods: Pulmonary specimens included 34 surgical lung biopsies, histologically classified as UIP, from patients clinically diagnosed with IPF. Fibroblast telomerase expression, interstitial myofibroblast $\alpha$-AMS expression and IL-4 (interleukin 4), TGF- $\beta$ (transforming growth factor beta) and bFGF (basic fibroblast growth factor) tissue expression were evaluated by immunohistochemistry and quantified in collapsed (COL), mural fibrosis (MF) and honeycombing areas (HC). Results: Telomerase expression was significantly higher in $\mathrm{COL}$ areas than in MF and $\mathrm{HC}$ areas. The same was observed for b-FGF. Interleukin- 4 and $\alpha$-AMS expression were significantly higher in MF areas. TGF- $\beta$ expression ws higher in $\mathrm{COL}$ and $\mathrm{HC}$ areas. We observed a positive correlation between telomerase and bFGF expression in COL, MF and $\mathrm{HC}$ areas. The same was noted for $\alpha$-AMS and IL-4. In MF areas, a negative correlation between IL-4 and b-FGF was obtained and TGF- $\beta$ tended to positively

COMU 2010 - Prêmio Oswaldo Cruz - Área Básica.

1. Acadêmico do $6^{\circ}$ ano de Medicina da Faculdade de Medicina da Universidade de São Paulo (FMUSP).

2. Médico Assistente do Departamento de Patologia da Faculdade de Medicina da Universidade de São Paulo (FMUSP).

3. Professor Emérito do Departamento de Pneumologia da Faculdade de Medicina da Universidade de São Paulo (FMUSP).

4. Professora Associado do Departamento de Patologia da Faculdade de Medicina da Universidade de São Paulo (FMUSP).

Endereço para correspondência: Faculdade de Medicina da Universidade de São Paulo (FMUSP). Av. Dr. Arnaldo 455, sala 1143, CEP 01296-903, São Paulo, SP. e-mail: daniel_waisberg@ hotmail.com 
FM e FV. O mesmo ocorreu com a expressão de $\alpha$-AMS e IL4. Nas áreas de FM, houve uma correlação negativa entre IL-4 e bFGF, e TGF- $\beta$ apresentou tendência a associação positiva com $\alpha$-AMS. Análise multivariada revelou que a expressão de IL-4 e $\alpha$-AMS nas áreas de FM são indicadores independentes de menor sobrevida em modelo estatístico significante incluindo idade, tabagismoe FVC (capacidade vital forçada). Pacientes com expressão de IL-4 menor que $13,5 \%$ nas áreas de FM apresentaram melhor sobrevida. O mesmo foi observado para expressão de $\alpha$-AMS menor que $8,5 \%$. Conclusão: Fibroblastos, com capacidade multiplicativa caracterizada pela expressão de telomerase e de bFGF tecidual, tendem a predominar no estágio precoce de remodelamento da FPI/PIU; enquanto a diferenciação de miofibroblastos, caracterizada pela expressão de $\alpha$-AMS e IL-4, parece ser responsável pelo estágio tardio da resposta fibrótica. A sobrevida dos pacientes parece ser influenciada por tais processos.

DESCRITORES: Telomerase; Fibroblastos; Fibrose pulmonar idopática; Pneumonia intersticial usual; Prognóstico.

\section{INTRODUÇÃO}

A fibrose pulmonar idiopática (FPI) consiste em uma pneumonia intersticial fibrosante de etiologia desconhecida e conseqüências devastadoras. Tipicamente, é caracterizada por ter deposição excessiva de colágeno, aumento de sua prevalência com a idade e remodelamento irreversível do parênquima pulmonar $^{1}$. O exame histopatológico revela um padrão de pneumonia intersticial usual (PIU) com áreas de fibrose mural variável, intercaladas com parênquima pulmonar normal, colapsado ou com faveolamento ${ }^{2}$. Apesar de não se ter definida a etiologia da FPI, existe a hipótese de que fibroblastos e miofibroblastos possam representar células efetoras fundamentais na sua patogênese $e^{3,4}$. Dependendo de estímulo ambiental específico, fibroblastos podem se diferenciar em miofibroblastos ou proliferar, resultando em focos fibroblásticos. Estes são considerados como sendo o local principal de remodelamento tecidual, com síntese ativa de matriz extracelular (MEC), colágeno e fibronectina ${ }^{5}$.

Nesse sentido, o fator de crescimento transformador $\beta 1$ (TGF- $\beta 1$ ) é um dos mais potentes indutores de deposição de colágeno, sendo fundamental seu efeito fibrogênico na cicatrização e reparo tecidual. A função principal do TGF- $\beta 1$ envolve a transição de fibroblastos quiescentes para um fenótipo ativado, denominado miofibroblastos, em que temos como conseqüências a produção de MEC e contração da ferida ${ }^{6}$. A interleucina-4 (IL-4) tem se mostrado um potente indutor da produção de TGF- $\beta 1$ no parênquima pulmonar, acarretando aumento da síntese de $M E C^{7,8}$, bem como tem se mostrado um potente fator quimiotático na movimentação de miofibroblastos ${ }^{9}$. Quando diferenciados e ativados, correlate with $\alpha$-AMS. In multivariate analysis, IL-4 tissue and $\alpha$-AMS myofibroblast expression in MF areas were independently predictive of mortality in a statistically significant model including age, tobacco use and FVC (full vital capacity). Patients with IL-4 expression lower than $13.5 \%$ in MF areas had better survival. The same was noted for $\alpha$-AMS expression lower than $8.5 \%$. Conclusion: Fibroblast multiplicative capacity, characterized by telomerase expression and associated with bFGF tissue expression, seems to predominate in the early remodeling process of IPF/UIP, whereas myofibroblast differentiation, characterized by alpha-smooth muscle actin expression and associated with IL-4 tissue expression, seems to lead to the later fibrotic response. Patient survival seems to be influenced by the intensity of this process.

KEYWORDS: Telomerase; Fibroblasts; Idiopathic pulmonary fibrosis; Usual interstitial pneumonia; Prognosis.

os miofibroblastos são caracterizados pela expressão de $\alpha$-actina de músculo liso $(\alpha \text {-AMS })^{10}$. Um aspecto central nas hipóteses acerca da patogênese da FPI é a persistência de miofibroblastos ativados que continuam a sintetizar MEC.

A telomerase é uma polimerase especializada que adiciona sequências repetidas de nucleotídeos às terminações dos cromossomos usando seu componente intrínseco de RNA como fita molde, compensando, assim, o encurtamento dos telômeros que ocorre normalmente a cada divisão celular ${ }^{11-13}$. A telomerase, dessa forma, mostrou-se essencial para a proliferação celular ilimitada e imortalidade ${ }^{14}$.

Evidências de ativação da telomerase na transformação celular sugerem sua importância provável na carcinogênese. Entretanto, existem também evidências indicando aumento da expressão da telomerase no microambiente inflamatório tecidual ${ }^{15}$. Dados mais recentes apontam para uma limitação da replicação de pneumócitos tipo II devido à expressão anormal da telomerase e de fatores relacionados à apoptose no remodelamento precoce da $\mathrm{PIU}^{16}$. A telomerase pode ter sua expressão aumentada no dano tecidual induzido pela sílica ${ }^{17}$ e em culturas de sinoviócitos de pacientes com artrite reumatóide ${ }^{18}$. $\mathrm{O}$ último estudo também indica que o aumento da expressão de telomerase nos sinoviócitos pode ocorrer devido ao fator de crescimento básico de fibroblastos (bFGF). A lesão pulmonar induzida por bleomicina e a fibrose também são conhecidas como indutoras da atividade da telomerase nos tecidos pulmonares afetados e em fibroblastos pulmonares isolados ${ }^{19}$, que ocorre via maior expressão de bFGF.

Fibroblastos isolados de pulmões com fibrose 
induzida por bleomicina em camundongos mostram aumento intrínseco de sua capacidade proliferativa e são capazes de se diferenciar em miofibroblastos que expressam $\alpha$-AMS, processo este acompanhado por menor expressão de telomerase / bFGF e maior expressão de IL-4 e TGF- $\beta^{11,19-21}$. A indução da atividade da telomerase está associada com aumento do número de fibroblastos em fibrose pulmonar induzida por bleomicina, e os padrões de expressão de genes codificadores de telomerase indicam que a atividade da telomerase está primariamente localizada no fibroblasto, presumivelmente antes de sua diferenciação em miofibroblastos ou em outros tipos celulares. Estes achados sugerem que a população de fibroblastos do tecido pulmonar alterado pode conter células que expressam telomerase (e, portanto, com vida útil estendida), contribuindo para ao aumento do número de fibroblastos pulmonares observado $^{19}$. Contudo, o mecanismo de indução e regulação da expressão da telomerase no contexto da PIU / FPI ainda não foi estudado.

À luz dos achados prévios sugerindo uma interação entre telomerase e proliferação de fibroblastos, o objetivo do presente trabalho é avaliar: a relação entre a expressão de citocinas pró-fibróticas (TGF- $\beta$, IL-4, bFGF) e a expressão de telomerase em fibroblastos e $\alpha$-AMS em miofibroblastos nas diversas fases do remodelamento na PIU. Hipotetizamos que na PIU, uma intensa pneumonia intersticial fibrosante crônica, a indução da atividade da telomerase nos fibroblastos pode estar associada a diferentes citocinas, com propriedades inflamatórias e fibrogências, dependendo do estágio do remodelamento.

\section{MATERIAIS E MÉTODOS}

O protocolo deste estudo foi revisado e aprovado pela Comissão de Ética para Análise de Projetos de Pesquisa de nossa instituição (CAPPesq 0731/07).

\section{Casuística}

O estudo incluiu 34 biópsias cirúrgicas de pulmão de pacientes com fibrose pulmonar idiopática (FPI), 19 homens e 15 mulheres, com mediana de idade de 69 anos (variação de 49 a 77 anos), de acordo com os critérios delineados no American Thoracic Society/European Respiratory Society International Multidisciplinary Consensus Classification of the Idiopathic Interstitial Pneumonias ${ }^{22}$. Pneumonia intersticial usual (PIU), o padrão histológico da FPI, foi caracterizada pela distribuição desigual da injúria ao parênquima pulmonar subpleural e paraseptal (Figura 1A). A heterogeneidade temporal foi vista em pequeno aumento, com alternância de áreas de parênquima pulmonar normal e colapso alveolar (Figura 1B), áreas de fibrose mural, com tecido septal fibromixóide (Figura 1C), e faveolamento pulmonar (Figura 1D).

Os prontuários dos pacientes envolvidos foram estudados retrospectivamente, a fim de se estabelecer data de último contato, os valores de prova de função pulmonar e a presença ou ausência de tabagismo. A sobrevida foi calculada como o tempo entre a realização de biópsia pulmonar e a data de último contato ou data de óbito. Procuramos atualizar a data de último contato para aqueles pacientes cuja data era anterior a junho de 2010. Aqueles em que isso não foi possível foram considerados falecidos, sendo a data de último contato considerada como a de óbito. As provas de função pulmonar utilizadas foram aquelas mais próximas da data de realização de biópsia pulmonar.

\section{Análise imunohistoquímica}

A análise imunohistoquímica foi realizada utilizando kits comercialmente disponíveis para caracterizar a presença de TGF- $\beta$ (polyclonal rabbit, cat Sc-146, 1:1500 dilution, Santa Cruz, Biotechnology Inc, CA, USA), IL-4 (polyclonal rabbit, cat 7919, 1:50 dilution, Santa Cruz Biotechnology Inc, CA, USA), $\alpha$-SMA (monoclonal mouse, cat MO 851, clone clone 1A4, 1:500 dilution, Dakocitom Glostrup Denmark Sigma, St Louis, Mo), telomerase (policlonal rabbit, cat 582005, 1:500 dilution, Calbiochem U.S. EMD Biosciences Inc., La Jolla, CA, US, Affiliate of Merck KGA, Darmstadt, Germany) e bFGF (anti-FGF- 2/ basic FGF, clone bFM-2, cat 05-118, 1:100 dilution, upstate, Temecula, CA). Em seguida, as seções foram desparafinizadas e rehidratadas com solução salina TBS (Tris-buffered, $0.0005 \mathrm{~mol} / \mathrm{L}$ Tris and $0.15 \mathrm{M} \mathrm{NaCl}[\mathrm{pH} 7.6])$ por 10 minutos. A peroxidase endógena foi bloqueada com $3 \%$ de peróxido de hidrogênio por 5 minutos. A recuperação do antígeno foi feita com 10mM de tampão de citrato, $\mathrm{pH}=6 \mathrm{e}$ de tripsina para IL-4. Em seguida, as seções foram lavadas em solução salina TBS e incubadas com anticorpos primários na diluição apropriada durante a noite, entre $4^{\circ}$ a $8^{\circ} \mathrm{C}$. O kit Novolink (cod RE 7280$\mathrm{K}$, MaxPolymer Detector System, Leika Biosystems Novocastle St, UK) foi usado como anticorpo secundário. A reação da peroxidase foi desenvolvida usando 3,3-diaminobenzidina tetrahidrocloreto $(0,25$ $\mathrm{mg}$ dissolvidos em $1 \mathrm{~mL}$ de peróxido de hidrogênio $0,02 \%)$ por 3 minutos. 


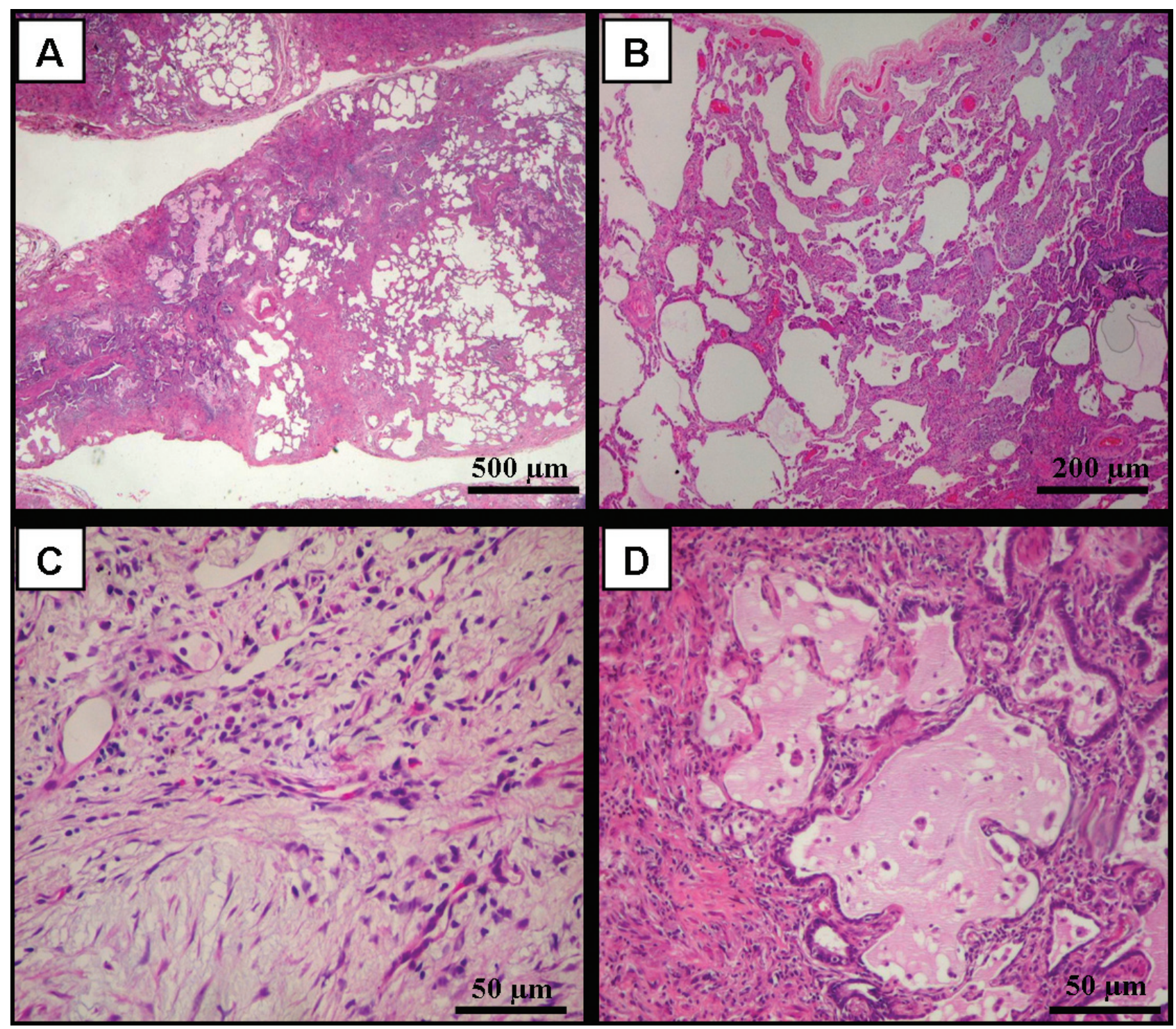

FIGURA 1. Padrão histológico da pneumonia intersticial usual. A- Distribuição desigual da injúria ao parênquima pulmonar subpleural e paraseptal. B - Heterogeneidade temporal, com alternância de áreas de parênquima pulmonar normal e colapso alveolar. C - Área de fibrose mural, com tecido septal fibromixóide. D- Faveolamento pulmonar

\section{Morfometria}

A expressão de TGF- $\beta$, IL-4, $\alpha$-AMS, telomerase e bFGF foi avaliada em 10 campos pela técnica de contagem de pontos nas áreas colapsadas, de fibrose mural e de faveolamento na PIU, usando um retículo de 100 pontos com área conhecida (62500 $\mu \mathrm{m} 2$ a um aumento de 400x) acoplada à ocular do microscópio ${ }^{23}$. A um aumento de 400x, o número de pontos sobre o parênquima pulmonar foi contado. Após isso, foi quantificado o número de pontos sobre células positivas para os diversos marcadores em cada uma das áreas do tecido pulmonar. A relação de células positivas foi determinada pela divisão deste último número pelo de pontos totais sobre o parênquima pulmonar, em cada campo, para cada marcador. Os resultados finais foram expressos na forma de porcentagem. Para cada área, 10 campos foram quantificados, sendo o resultado final de expressão de um determinado marcador em uma dada área obtido por meio de média aritmética. Foram consideradas células positivas para TGF- $\beta$, IL-4 e bFGF todas aquelas imunocoradas, representando a expressão tecidual desses marcadores. Para telomerase, somente células presentes no interstício alveolar e morfologicamente 
Waisberg et al. Aumento na expressão de telomerase por fibroblastos.

semelhantes a fibroblastos foram consideradas positivas. Para $\alpha$-AMS, apenas células imunocoradas presentes no interstício foram consideradas positivas, desde que não apresentassem morfologia de células muscular lisa ou compusessem a parede de vasos sanguíneos. Comparações interobservador foram realizadas em $20 \%$ dos cortes por dois observadores (D.R.W e V.L.C.). O coeficiente de variação para o erro interobservador de contagem de células foi $<5 \%$.

\section{Análise estatística}

Um teste-t independente foi usado para testar a relação entre variáveis contínuas, após o teste de Kolmogorov-Smirnov ter classificado as variáveis como paramétricas. Para verificar a associação entre a expressão de TGF- $\beta$, IL-4, $\alpha$-AMS, telomerase e bFGF, o teste de Pearson foi usado. A análise estatística foi realizada com o software SPSS (SPSS, Inc, v.18.0, Chicago, IL, 2009). A significância estatística foi definida como $p \leq 0,05$.

\section{RESULTADOS}

\section{Expressão tecidual de TGF- $\beta$}

A Figura 2 mostra as células que expressaram TGF- $\beta$, coradas de acastanhado pela reação de imunohistoquímica. A expressão tecidual desse marcador foi avaliada no parênquima pulmonar normal, nas áreas colapsadas (Figura 2A), nas áreas de fibrose mural (Figura 2B) e de faveolamento (Figura 2C) da PIU.

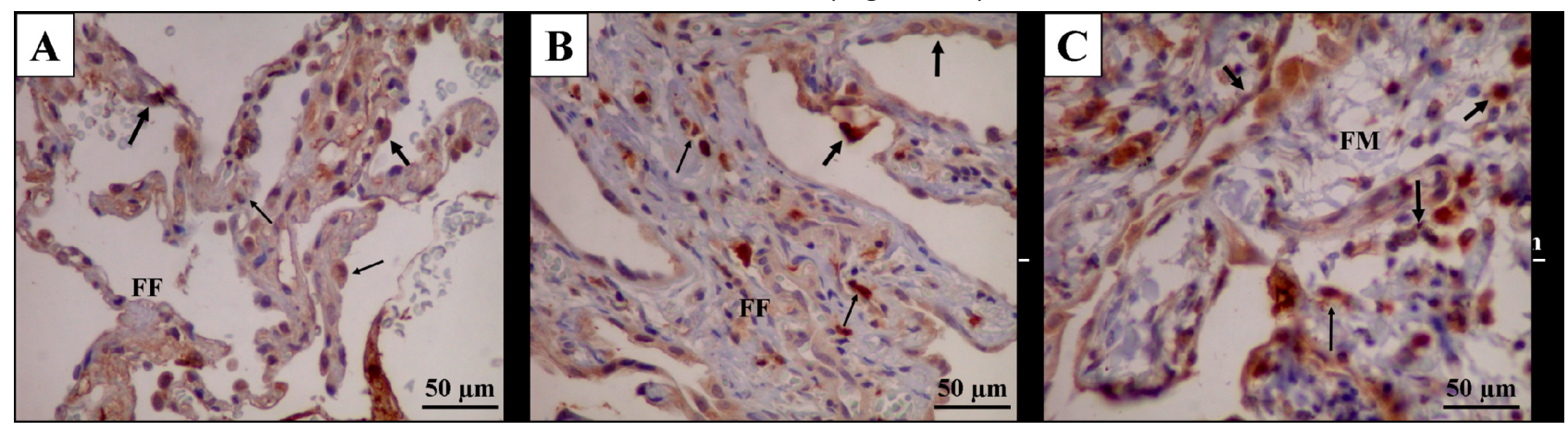

FIGURA 2. Reação de imunohistoquímica corando de acastanhado as células que expressaram TGF- $\beta$ nas áreas colapsadas (A), fibrose mural (B) e faveolamento (C). As setas apontam para células tipicamente positivas. FF, foco fibroblástico; FM, fibrose mural; HC, faveolamento (honeycombing)

A análise quantitativa revelou que a expressão tecidual de TGF- $\beta$ foi significativamente menor nas áreas normais $(9,46 \pm 2,04 \%)$ em relação às áreas colapsadas $(19,63 \pm 5,70 \%, p<0,01)$, de fibrose mural $(16,48 \pm 4,11 \%, p<0,01)$ e de faveolamento $(17,08 \pm 4,52 \%, p<0,01)$. A expressão nas áreas de colapso foi significantemente maior que nas áreas de fibrose mural $(p=0,03)$, mas estatisticamente semelhante a das áreas de faveolamento $(p=0,51)$. A expressão nas áreas de fibrose mural foi menor que nas de faveolamento $(p=0,042)$.

\section{Expressão tecidual de IL-4}

A expressão tecidual de IL-4 pela imunohistoquímica pode ser observada na Figura 3: áreas colapsadas (Figura $3 \mathrm{~A}$ ), áreas de fibrose mural (Figura 3B) e áreas de faveolamento (Figura 3C). A expressão dessa interleucina foi maior nas áreas de fibrose mural. Não houve expressão significativa no parênquima pulmonar normal.

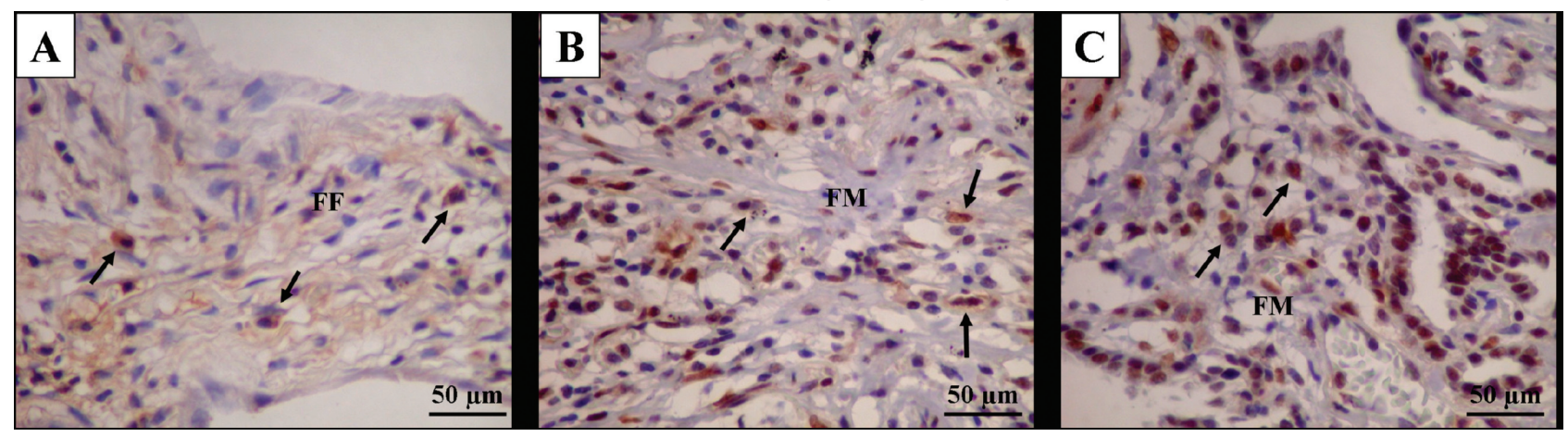

FIGURA 3. Expressão tecidual de IL-4 pela imunohistoquímica em áreas colapsadas (A), áreas de fibrose mural (B) e áreas de faveolamento (C). A expressão foi maior nas áreas de fibrose mural. Não houve expressão significativa no parênquima pulmonar normal. As setas apontam para células tipicamente positivas. FF, foco fibroblástico; FM, fibrose mural; HC, faveolamento (honeycombing) 
À análise quantitativa, a expressão de IL-4 foi significativamente maior $(p<0,01)$ nas regiões de fibrose mural da PIU $(14,03 \pm 6,00 \%$ ), quando comparada à expressão nas regiões colapsadas $(3,27 \pm 5,48 \%)$ e de faveolamento $(7,91 \pm 4,04 \%)$. A expressão nas áreas de faveolamento também foi maior que nas áreas colapsadas $(p<0.01)$.

\section{Expressão tecidual de bFGF}

A Figura 4 mostra as células que expressaram bFGF, coradas de acastanhado pela reação de imunohistoquímica. A expressão desse marcador foi avaliada no parênquima pulmonar normal, nas áreas colapsadas (Figura 4A), nas áreas de fibrose mural (Figura 4B) e de faveolamento (Figura 4C) da PIU.

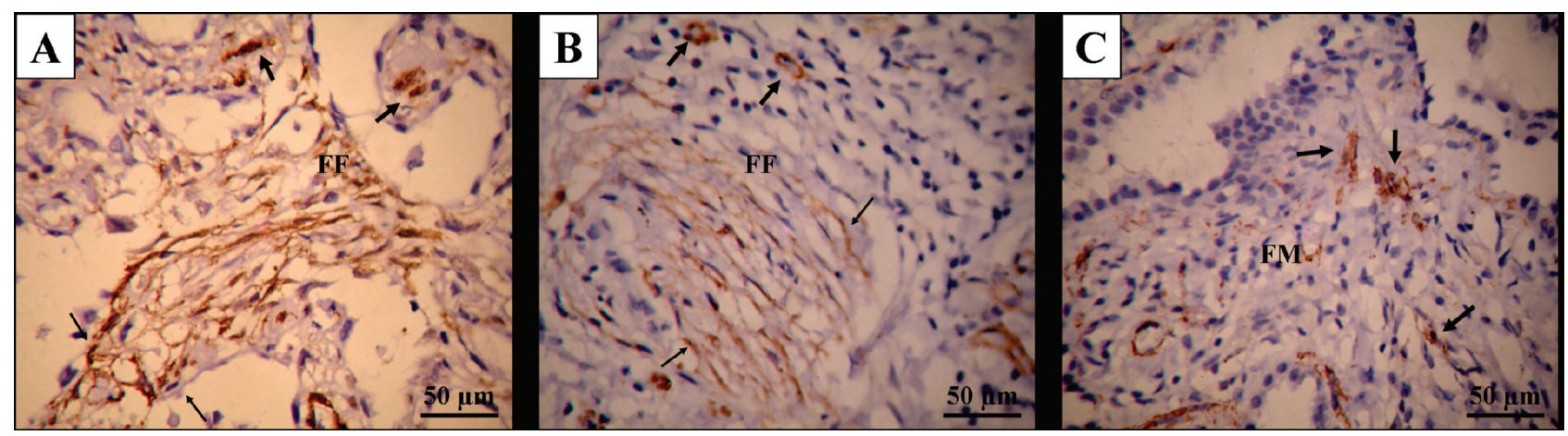

FIGURA 4. Expressão tecidual de bFGF em áreas de colapso (A), de fibrose mural (B) e de faveolamento (C) da PIU. Apenas o parênquima normal não apresentou expressão relevante de bFGF. A expressão foi maior nas áreas de colapso. As setas apontam para células tipicamente positivas. FF, foco fibroblástico; FM, fibrose mural; HC, faveolamento (honeycombing)

Na análise quantitativa, pudemos verificar que a expressão tecidual de bFGF foi significativamente maior $(p<0,01)$ nas áreas colapsadas do parênquima pulmonar $(15,50 \pm 4,99 \%)$ do que nas áreas do parênquima considerado normal $(10,57 \pm 6,24 \%)$, de fibrose mural $(11,62 \pm 3,62 \%)$ e de faveolamento $(12,21 \pm 4,68 \%)$, que foram estatisticamente semelhantes entre si $(p=0,37$ para normal $x$ fibrose mural; $p=0,26$ para normal $x$ faveolamento $e$ $p=0,21$ fibrose mural $x$ faveolamento).

\section{Expressão de telomerase por fibroblastos}

A Figura 5 demonstra a expressão da telomerase através da reação de imunohistoquímica. Foram representadas as áreas de colapso (Figura $5 \mathrm{~A}$ ), de fibrose mural (Figura 5B) e de faveolamento (Figura 5C. À exceção do parênquima pulmonar normal, as demais áreas de PIU tiveram expressão significativa de telomerase por fibroblastos.

A análise quantitativa revelou que a expressão da telomerase por fibroblastos foi significativamente maior $(p<0,01)$ nas áreas colapsadas $(6,76 \pm 1,31 \%)$ do que nas áreas de fibrose mural $(5,26 \pm 1,13 \%)$ e de faveolamento $(5,01 \pm 1,14 \%)$, que foram semelhantes estatisticamente entre si $(p=0,65)$.

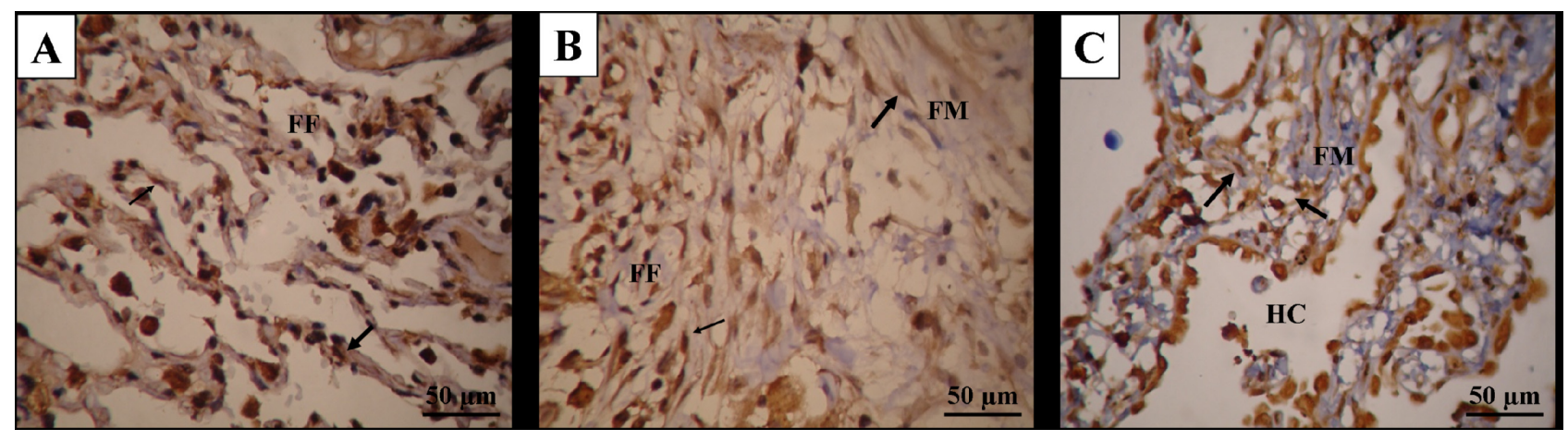

FIGURA 5. Expressão da telomerase por fibroblastos em áreas de colapso (A), de fibrose mural (B) e de faveolamento (C). Os maiores valores foram encontrados nas áreas de colapso. As setas apontam para células tipicamente positivas. FF, foco fibroblástico; FM, fibrose mural; HC, faveolamento (honeycombing) 
Waisberg et al. Aumento na expressão de telomerase por fibroblastos.

\section{Expressão de $\alpha$-AMS por miofibroblastos}

A Figura 6 apresenta a expressão de $\alpha$-AMS nas regiões de colapso (Figura $6 \mathrm{~A}$ ), de fibrose mural (Figura 6B) e de faveolamento (Figura 6C). A expressão de $\alpha$-AMS por miofibroblastos foi maior nas regiões de fibrose mural. O parênquima normal não expressou quantidade relevante de $\alpha$-SMA.

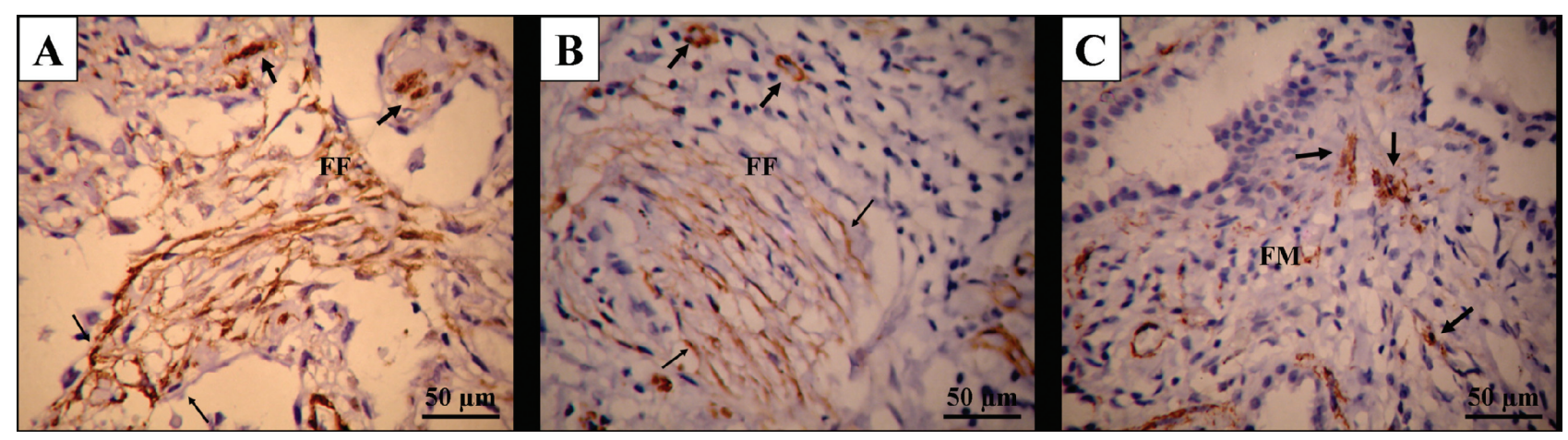

FIGURA 6. Expressão de $\alpha$-SMA por miofibroblastos nas regiões de colapso (A), de fibrose mural (B) e de faveolamento (C). A expressão de $\alpha$-SMA foi maior nas regiões de fibrose mural. O parênquima normal não expressou quantidade relevante de $\alpha$-SMA. As setas apontam para células tipicamente positivas. FF, foco fibroblástico; FM, fibrose mural; HC, faveolamento (honeycombing)

A análise quantitativa novamente confirmou o padrão de distribuição morfológica de $\alpha$-AMS. A expressão desse marcador foi significativamente maior $(p<0,01)$ nas regiões de fibrose mural $(9,02 \pm$ $2,92 \%)$, quando comparada à expressão nas regiões de colapso $(5,25 \pm 5,14 \%)$ e de faveolamento $(5,21 \pm$ $2,13 \%)$, que foram estatisticamente entre si $(p=0,78)$.

\section{Correlação entre variáveis morfométricas}

As associações significativas encontradas entre a expressão tecidual de TGF- $\beta$, IL-4 e bFGF, expressão de telomerase por fibroblastos e expressão de $\alpha$-AMS por miofibroblastos é mostrada na Tabela 1. Resumidamente, a expressão de bFGF e telomerase foram positivamente associadas nas três áreas estudadas, enquanto o mesmo foi observado para IL-4 e $\alpha$-AMS. Uma correlação negativa entre IL-4 e bFGF nas áreas de fibrose mural também foi observada. Uma tendência de correlação positiva entre TGF- $\beta$ e $\alpha$-AMS nas áreas de fibrose mural (coeficiente de correlação $=0,39 ; p=0,06$ ) também foi observada.

TABELA 1. Correlação entre variáveis morfométricas estudadas

\begin{tabular}{cccc}
\hline Variáveis & Áreas & Coeficiente de Correlação & $\mathbf{p}$ \\
\hline \multirow{2}{*}{ bFGF e telomerase } & Colapso & .41 & .03 \\
& Fibrose mural & .43 & .02 \\
& Faveolamento & .62 & .01 \\
$\alpha-\mathrm{AMS}$ e IL-4 & Colapso & .39 & .05 \\
& Fibrose mural & .60 & .01 \\
$\mathrm{IL}-4 \mathrm{e}$ bFGF & Faveolamento & .62 & .01 \\
\hline
\end{tabular}




\section{Análise da sobrevida e variáveis clínicas}

O estudo da sobrevida através da análise de multivariada de Cox é mostrado na Tabela 2. Um total de $18(52,9 \%)$ pacientes eram tabagistas. As médias de $\operatorname{VEF}_{1}$ (volume expiratório forçado no $1^{\circ}$ segundo) e de FVC (capacidade vital forçada) para a população deste estudo foram de $69,76 \% \pm 21,66$ e $66,33 \% \pm$ 12,54 do valor predito, respectivamente, resultando em uma média de $\mathrm{VEF}_{1} / \mathrm{FVC}$ de 109,72 \pm 14,02. Cerca de $64,7 \%(n=22)$ pacientes faleceram, ao passo que $26,4 \%$ ( $n=9)$ estavam vivos ao término do seguimento. Perdemos o contato de três pacientes $(8,8 \%)$, considerados como falecidos na data de último contato. O tempo médio de sobrevida após o diagnóstico de FPI foi de 29,67 $\pm 20,16$ meses. Quatro variáveis estiveram associadas com a sobrevida dos pacientes: idade, capacidade vital forçada, expressão de $\alpha$-AMS e IL-4 nas áreas de fibrose mural da PIU. A análise multivariada apontou como indicadores de menor sobrevida as seguintes condições: idade maior que 68 anos (OR =2,44, $p=0,03$ ), FVC (capacidade vital forçada) menor que $66 \%$ do valor predito (OR $=0,94 ; p=0,02)$ e expressão de $\alpha$-AMS e IL-4 em fibrose mural maior que $8,55 \%(O R=4,84 ; p=0,04)$ e $13,40 \%(O R=13,40 ; p=0,04)$, respectivamente. O gráfico relacionando sobrevida com o tempo de seguimento em meses de pacientes com FPI mostrou que aqueles com expressão, nas áreas de fibrose mural, de $\alpha$-SMA menor que 8,55\% e de IL4- menor que 13,9\% apresentaram maior sobrevida (Figura 7).

TABELA 2. Análise multivariada de Cox (coeficiente do modelo $=26,109 ;$ Chi-square $=13,741 ; p=0.01$ )

\begin{tabular}{|c|c|c|c|c|}
\hline & \multirow{2}{*}{ Odds Ratio } & \multirow{2}{*}{$p$} & \multicolumn{2}{|c|}{$95 \%$ IC } \\
\hline & & & Mínimo & Máximo \\
\hline $\begin{aligned} & \text { Idade } \\
> & 68 \text { anos }\end{aligned}$ & 2.44 & 0.03 & 0.35 & 16.77 \\
\hline Tabagismo & 0.86 & 0.868 & 0.16 & 4.55 \\
\hline $\begin{array}{c}\text { FVC } \\
>66 \%\end{array}$ & 0.94 & 0.02 & 0.86 & 1.03 \\
\hline $\begin{array}{c}\alpha \text {-AMS - fibrose mural } \\
>8.55 \%\end{array}$ & 4.84 & 0.04 & 0.57 & 41.03 \\
\hline $\begin{array}{c}\text { IL-4 - fibrose mural } \\
>13.40 \%\end{array}$ & 10.10 & 0.04 & 1.08 & 94.43 \\
\hline
\end{tabular}

FVC, capacidade vital forçada; IC, intervalo de confiança
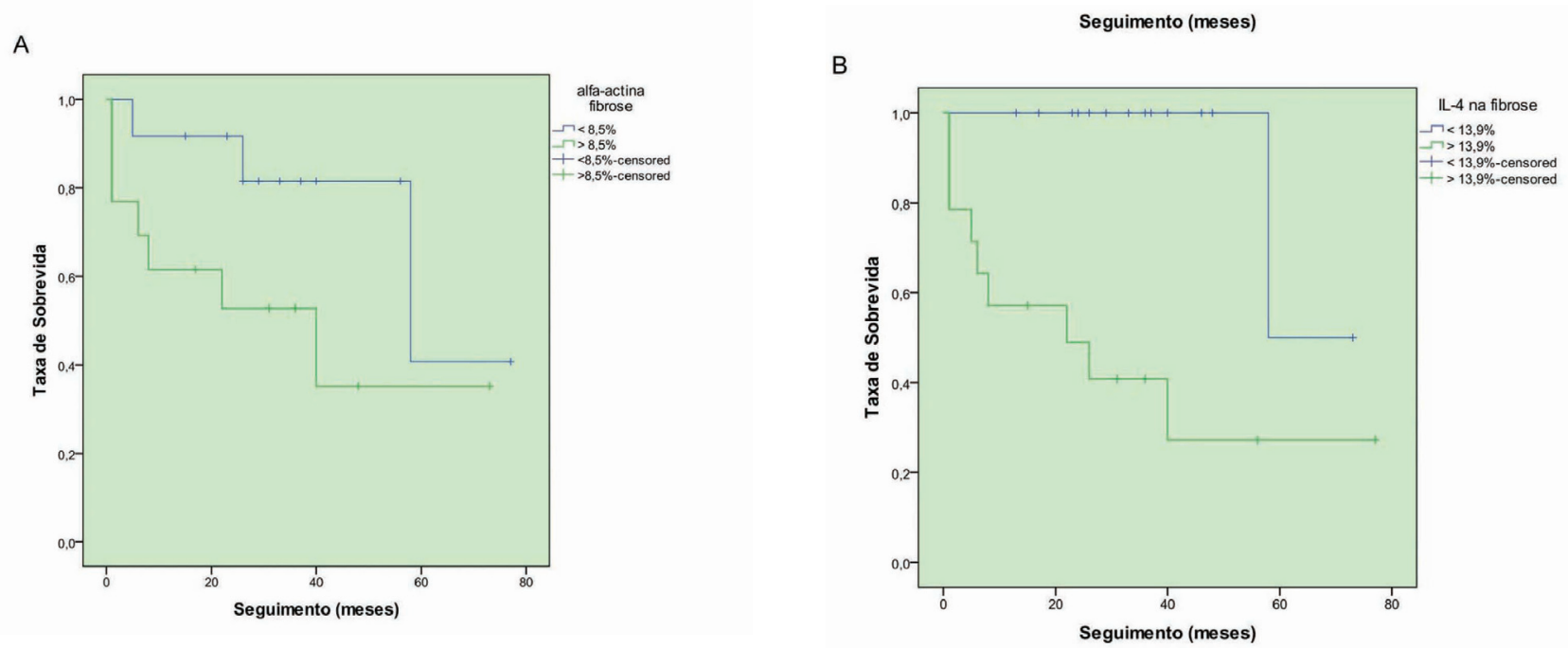

FIGURA 7. A - Análise multivariada de Cox para probabilidade de sobrevida versus seguimento em meses. O grupo com expressão de $\alpha$-AMS em área de fibrose mural $>8,55 \%$ aparece como a curva inferior, enquanto aquele com expressão $<8.55 \%$ aparece na curva superior. B - Idem para expressão de IL-4 > 13,9\% (curva inferior) e < 13,9\% (curva superior) 
Waisberg et al. Aumento na expressão de telomerase por fibroblastos.

\section{DISCUSSÃO}

Investigamos a participação da telomerase e de fatores responsáveis por sua ativação ou supressão no processo fibrótico encontrado em cortes pulmonares obtidos por biópsia cirúrgica de pacientes com padrão histológico de pneumonia intersticial usual (PIU). Especificamente, avaliamos a expressão da telomerase em fibroblastos e de $\alpha$-AMS em miofibroblastos de diversas áreas de PIU para verificar possíveis associações com a expressão tecidual de TGF- $\beta$, IL-4 e bFGF. Os resultados obtidos representam a possibilidade de introduzirmos novos marcadores ou novos protocolos para o tratamento de pacientes com fibrose pulmonar idiopática (FPI). $A$ expressão tecidual de TGF- $\beta$ foi maior nas áreas colapsadas e de faveolamento. A expressão tecidual de IL-4 e de $\alpha$-SMA por miofibroblastos foi maior nas áreas de fibrose mural, enquanto a expressão tecidual de bFGF e de telomerase por fibroblastos o foi nas áreas de colapso. Além disso, a expressão de $\alpha$-SMA esteve diretamente associada à de IL-4 nas três áreas estudadas. $O$ mesmo foi observado para bFGF e telomerase.

A telomerase é uma multi-subunidade ribonucleoprotéica específica que adiciona a seqüência TTAGGG às terminações dos cromossomos, usando seu componente intrínseco de RNA como fita molde, compensando, dessa forma, a perda dos telômeros que naturalmente ocorre a cada divisão celular ${ }^{12,13}$. Demonstrou-se ter a telomerase papel essencial na replicação celular ilimitada ${ }^{14}$. Evidências de que a telomerase está ativada em células transformadas e tumores sugerem sua provável função na carcinogênese. Contudo, estudos recentes indicam que a telomerase também é expressa em células presentes em processos inflamatórios, assim como em tecidos normais como baço, timo e testículos ${ }^{12,24,25}$. Tecido mamário e amostras de pele de ratos exibem baixos níveis de telomerase, que podem aumentar quando as células são isoladas e crescem em meios de cultura ${ }^{26}$. Dados mais recentes mostram que a atividade da telomerase pode ser induzida em modelos de injúria pulmonar por sílica ${ }^{17}$ e em culturas de sinoviócitos de pacientes com artrite reumatóide ${ }^{18}$. Este último estudo também indica que a maior expressão da telomerase em sinoviócitos pode ser induzida por bFGF.

Nosso estudo revela que a expressão da telomerase e de bFGF aumenta a replicação de fibroblastos no estágio precoce de remodelamento na FPI/PIU. Tal achado é apoiado pelo trabalho de Nozaki et al. ${ }^{19}$, que demonstrou que a fibrose pulmonar induzida por bleomicina também é caracterizada por atividade aumentada da telomerase no tecido pulmonar e nos fibroblastos isolados desse tecido. Essa atividade aumentada ocorre, porém, apenas durante o período de ativa fibrose e está localizada primariamente em fibroblastos e miofibroblastos ${ }^{19}$. A atividade também declina in vitro com o passar do tempo, o que está associado à diferenciação em miofibroblastos. Estes estudos sugerem que a indução da telomerase em fibroblastos pode ser importante na resposta inflamatória crônica e fibrose. Os fatores responsáveis pela regulação da atividade da telomerase nesses tecidos são desconhecidos.

Um outro estudo, feito por Liu et al. ${ }^{11}$, examinou os efeitos da deficiência da transcriptase reversa da telomerase (TERT) em um modelo de lesão pulmonar em camundongos e demonstrou que tal deficiência resultou em redução da fibrose pulmonar após exposição a bleomicina. Este processo foi acompanhado por redução significante da expressão pulmonar de $\alpha$-SMA, um marcador de diferenciação de miofibroblastos. Além disso, fibroblastos pulmonares isolados de camundongos tratados com bleomicina e TERT-deficientes mostraram decréscimo significativo na proliferação e aumento da taxa de apoptose, comparando com células isoladas de camundongos controle. O transplante de medula óssea de camundongos controle naqueles TERT-deficientes restaurou a atividade de telomerase pulmonar induzida por lesão de bleomicina e fibrose a níves semelhantes dos controles. Por outro lado, o transplante de medula óssea dos camundongos TERT-deficientes para receptores controles resultou na redução de atividade de telomerase e fibrose. Estes achados sugerem que a indução da atividade da telomerase em modelos de injúria pulmonar pode ser causada por células derivadas da medula óssea, as quais devem ter um papel importante na fibrose pulmonar. Além disso, a indução da TERT está associada a um aumento da sobrevida de fibroblastos, que favorecem o desenvolvimento de fibrose ao invés de promover a resolução do processo de injúria ao pulmão.

Nosso estudo também sugere que $\alpha$-AMS e IL-4 estão restritos ao remodelamento tardio da FIP/PIU. A disposição espacial da expressão da telomerase e de bFGF - nas áreas de colapso alveolar, dentro de focos de fibroblásticos - alicerçam a hipótese de que a lesão epitelial e ativação de fibroblastos são cruciais na patogênese da FPI. Além disso, a presença de $\alpha$-SMA e IL-4 em áreas sob injúria contínua e fibrose na FPI indica uma potencial função na modificação da matriz extracelular, por meio de uma atividade colagenolítica ${ }^{27}$.

Hipóteses acerca da etiologia da progressão do processo fibrótico enfocaram aberrações no remodelamento tecidual envolvendo lesão epitelial e/ 
ou ativação de fibroblastos ${ }^{28}$. Estudos que avaliaram a reparação tecidual sem formação de cicatriz em tecidos fetais sugerem que tal processo decorra da presença de fenótipos específicos de células mesenquimais. Estes estudos identificaram miofibroblastos que estão ausentes durante o período em que a reparação tecidual ocorre sem formação de cicatriz, mas que aparecem posteriormente na gestação, permitindo a formação da cicatriz ${ }^{29}$. A este respeito, é considerado que fibroblastos funcionalmente heterogêneos nas áreas de injúria tecidual contribuam para a produção de matriz. Estudos prévios usando hibridização in situ identificaram miofibroblastos como o principal tipo celular responsável pela síntese de colágeno na FPI ${ }^{30}$. Miofibroblastos, caracterizados por sua expressão de $\alpha$-AMS, são conhecidos por estarem transitoriamente presentes durante a reparação tecidual normal e de forma mais prolongada durante a fibrose ${ }^{31}$. Porém, a expressão de $\alpha$-AMS em miofibroblastos nem sempre se correlaciona com a síntese de precursores de colágeno e desenvolvimento de fibrose ${ }^{32-34}$.

Portanto, consideramos que a expressão da telomerase está aumentada em fibroblastos submetidos a injúrias, levando a aumento da capacidade replicativa e consequente aumento do número destas células. Este fenômeno pode representar o estágio inicial do remodelamento associado à expansão de fibroblastos ${ }^{35}$. A inibição da

\section{REFERÊNCIAS}

1. Gross TJ, Hunninghake GW. Idiopathic pulmonary fibrosis. N Engl J Med 2001;345:517-25. Raghu G, Weycker D, Edelsberg J, Bradford WZ, Oster G. Incidence and prevalence of idiopathic pulmonary fibrosis. Am J Respir Crit Care Med. 2006;174:810-6.

2. Katzenstein AA, Myers JL. Idiopathic pulmonary fibrosis: clinical relevance of pathological classification. Am J Respir Crit Care Med. 1998;157:1301-9.

3. Uhal BD, Joshi I, True AL, et al. Fibroblasts isolated after fibrotic lung injury induce apoptosis of alveolar epithelial cells in vitro. Am J Physiol. 1995;269:L819-L828.

4. Ramos C, Montano M, Garcia-Alvarez J, et al. Fibroblasts from idiopathic pulmonary fibrosis and normal lungs differ in growth rate, apoptosis, and tissue inhibitor of metalloproteinases expression. Am J Respir Cell Mol Biol. 2001;24:591-8.

5. Elias JA, Zitnik RJ, Ray P. Fibroblast immune-effector function. In: Phipps RP, editor. Pulmonary fibroblast heterogeneity. Boca Raton: CRC Press; 1992. p.295322.

6. Desmouliere A, Geinoz A, Gabbiani F, Gabbiani G. Transforming growth factor-b1 induces a-smooth muscle actin expression in granulation tissue myofibroblasts and in quiescent and growing cultured fibroblasts. $J$ Cell Biol. 1993;122:103.

7. Hogaboam CM, Bone-Larson CL, Lipinski S, Lukacs atividade da telomerase, induzida por IL-4 e TGF- $\beta$, poderia resultar na diferenciação de fibroblastos em miofibroblastos, células com maior capacidade de síntese de matriz extracelular, e que estariam presentes no estágio tardio do remodelamento ou na fibrose estabelecida ${ }^{32}$.

Nosso estudo apresentou impacto clínico. A fim de determinar a relevância destes achados para a evolução dos pacientes, avaliamos a atividade da telomerase, a expressão de citocinas pró-fibróticas e de fatores de crescimento correlacionando-os com testes fisiológicos e com a sobrevida. Demonstramos, pois, através de análise multivariada, que pacientes com baixa FVC, alta expressão de $\alpha$-AMS e IL-4 mas áreas de fibrose mural apresentaram menor sobrevida.

Nossos achados revelam que o aumento na replicação de fibroblastos devido à maior expressão de bFGF e telomerase ocorre precocemente à ativação de miofibroblastos. O impulso à atividade destas últimas células, por sua vez, depende da expressão de $\alpha$-AMS e IL-4, já em um estágio mais tardio de remodelamento na pneumonia intersticial usual. Concluímos, portanto, que citocinas prófibróticas, telomerase e fatores de crescimento podem desempenhar a função de marcadores indiretos para a identificação de pacientes com maior risco de desenvolver fibrose pulmonar idiopática.

NW, Chensue SW, Strieter RM, et al. Differential monocyte chemoattractant protein-1 and chemokine receptor 2 expression by murine lung fibroblasts derived from Th1- and Th2-type pulmonary granuloma models. J Immunol. 1999;163:2193-8.

8. Makhluf HA, Stepniakowska J, Hoffman S, Smith E, LeRoy EC, Trojanowska M. IL-4 upregulates tenascin synthesis in scleroderma and healthy skin fibroblasts. J Invest. Dermatol. 1996;107:856-62.

9. Postlethwaite AE, Seyer JM. Fibroblast chemotaxis induction by human recombinant interleukin-4: identification by synthetic peptide analysis of two chemotactic domains residing in amino acid sequences 70-88 and 89-122. J. Clin Invest. 1991;87:2147-55.

10. Serini G, Gabbiani G. Mechanisms of myofibroblast activity and phenotypic modulation. Exp Cell Res. 1999;250:273-83.

11. Liu T, Nozaki Y, Phan SH. Regulation of telomerase activity in rat lung fibroblasts. Am J Respir Cell Mol Biol. 2002;48:534-40.

12. Greider CW. Telomere length regulation. Annu Rev Biochem. 1996;65:337-65.

13. Feng J, Funk WD, Wang SS, Weinrich SL, Avilion $\mathrm{AA}$, Chin $\mathrm{CP}$, et al. The RNA component of human telomerase. Science. 1995;269:1236-41.

14. Counter CM, Hirte HW, Bachetti S, Harley CB. 
Telomerase activity in human ovarian carcinoma. Proc Natl Acad Sci USA. 1994;91:2900-4.

15. Greider CW. Telomerase activity, cell proliferation, and cancer. Proc Natl Acad Sci USA. 1998;95:90-2.

16. Waisberg DR, Barbas-Filho JV, Parra ER MD, Fernezlian S, Carvalho CRR, Kairalla RA, Capelozzi VL. Abnormal expression of telomerase/apoptosis limits type II alveolar epithelial cell replication in the early remodeling of usual interstitial pneumonia/idiopathic pulmonary fibrosis. Human Pathol. 2010;41:385-91.

17. Kim JK, Lim Y, Kim KA, Seo MS, Kim JD, Lee KH, et al. Activation of telomerase by silica in rat lung. Toxicol Lett. 2000;111:263-70

18. Tsumuki $H$, Hasunuma $T$, Kobata $T$, Kato $T$, Uchida A, Nishioka K. Basic FGF-induced activation of telomerase in rheumatoid synoviocytes. Rheumatol Int. 2000;19:123-8.

19. Nozaki YT, Liu K, Hatano M, Gharaee-Kermani G, Phan $\mathrm{SH}$. Induction of telomerase activity in fibroblasts from bleomycin-injured lungs. Am J Respir Cell Mol Biol. 2000;23:460-5.

20. Phan SH, Varani J, Smith D. Rat lung fibroblast collagen metabolism in bleomycin-induced pulmonary fibrosis. J Clin Invest. 1985;76:241-7.

21. Zhang, HY, Gharaee-Kermani M, Zhang K, Karmiol $\mathrm{S}$, Phan SH. Lung fibroblast alpha-smooth muscle actin expression and contractile phenotype in bleomycin-induced pulmonary fibrosis. Am J Pathol. 1996;148:527-37.

22. American Thoracic Society. European Respiratory Society International Multidisciplinary Consensus Classification of the Idiopathic Interstitial Pneumonias. Am J Respir Crit Care Med. 2002;165:277-304.

23. Gundersen HJG, Bendtesen TF, Korbo L, Marcussen $\mathrm{N}$, Moller A, Nielsen K, et al. Some new, simple and efficient stereological methods and their use in pathological research and diagnosis. APMIS. 1988;96:379-94.

24. Hu BT, Insel RA. Up-regulation of telomerase in human B lymphocytes occurs independently of cellular proliferation and with expression of the telomerase catalytic subunit. Eur J Immunol. 1999;29:3745-53.

25. Funk WD, Wang CK, Shelton DN, Harley CB, Pagon GD, Hoeffler WK. Telomerase expression restores dermal integrity to in vitroaged fibroblast in a reconstituted skin model. Exp Cell Res. 2000;258:270-8
26. Chadeneau CP, Siegel CB, Harley W.J, Muller CF, Bacchetti F. Telomerase activity in normal and malignant murine tissues. Oncogene. 1995;11:893-8.

27. Park JE, Lenter MC, Zimmermann RN, Garin-Chesa P, Id LJ, Rettig WJ. Fibroblast activation protein, a dual specificity serine protease expressed in reactive human tumor stromal fibroblasts. J Biol Chem.1999;274:36512.

28. Selman M, King TE, Pardo A. Idiopathic pulmonary fibrosis: prevailing and evolving hypotheses about its pathogenesis andimplications for therapy. Ann Intern Med. 2001;134:136-51.

29. Estes JM, Vande Berg JS, Adzick NS, MacGillivray TE, Desmouliere A, Gabbiani G. Phenotypic and functional features of myofibroblasts in sheep fetal wounds. Differentiation. 1994;56:173-81.

30. Zhang K, Rekhter MD, Gordon D, Phan SH Myofibroblasts and their role in lung collagen gene expression during pulmonary fibrosis. A combined immunohistochemical and in situ hybridization study. Am J Pathol. 1994;145:114-25.

31. Sappino A, Schurch W, Gabbiani G. Differentiation repertoire of fibroblastic cells: expression of cytoskeletal proteins as marker of phenotypic modulation. Lab Invest. 1990;63:144-61.

32. Levy MT, McCauhan GW, Marinos G, Gorrell MD Intrahepatic expression of the hepatic stellate cell marker fibroblast activation protein correlates with the degree of fibrosis in hepatitis $\mathrm{C}$ virus infection. Liver. 2002;22:93-101.

33. Rubbia-brandt L, Mentha G, Desmouliere A, et al Hepatic stellate cells reversibly express alpha-smooth muscle actin during acute hepatic ischaemia. Transplant Proc. 1997;29:2390-5.

34. Iredale JP, Benyon RC, Pickering J, et al. Mechanisms of spontaneous resolution of rat liver fibrosis. Hepatic stellate cell apoptosis and reduced hepatic expression of metalloproteinase inhibitors. J Clin Invest. 1998;102:538-49.

35. Steinert S, Shay JW, Wright WE. Transient expression of human telomerase extends the life span of normal human fibroblasts. Biochem Biophys Res Commun. 2000;273:1095-8

Artigo premiado COMU: 18-23/10/10 